\title{
Teratogenic Effect of Sodium Phenytoin on Limb Development in Chick Embryos is Time-Mediated as Evident by Stereomicroscope
}

\author{
Hamd Binte Shahab Syed ', M. Yunus Khan ${ }^{2}$, Ruqqia Shafi Minhas ${ }^{3}$ \\ ${ }^{1}$ Assistant Professor, Department of Anatomy, CMH Institute of Medical Sciences, Multan \\ 2 Professor/Head, Department of Anatomy., CPSP Regional Center, Islamabad. \\ ${ }^{3}$ Assistant Professor, Department of Anatomy, Fazaia Medical College, Air University, Islamabad
}

\begin{abstract}
Background: Women suffering from seizure-related illnesses are prescribed phenytoin during pregnancy if the benefits outweigh the risks. But the teratogenic effect of this drug on limb development needs exploration. This study was designed to investigate the time-mediated teratogenic effect of sodium phenytoin on limb development of chick embryos on days 04 and 09 of development using the stereomicroscope and at the time of hatching.

Material and Methods: This randomized control trial comprised of two main groups of fertilized chicken eggs (Egyptian Fayoumi breed), control group A and experimental group B, each having 90 eggs. Each experimental egg was injected with sodium phenytoin ( $3.5 \mathrm{mg}$, teratogenic dose) just before incubation. Both the groups were divided into three subgroups each $A 1, A 2, \& A 3 / B 1, B 2$ \& B3. Stereomicroscope was used to observe the limb buds and cartilaginous elements in subgroups 1 and 2 on days 04 and 09 of development respectively. The embryos of subgroups 3 were assessed for gross limb deformities on hatching. Survival was noted in all the subgroups.

Results: The difference in limb bud size between experimental and controls was statistically insignificant. There was no variation or deformity in the bones. The experimental group 3 had gross limb defects on hatching and the difference in survival was also statistically significant in subgroups 3 .

Conclusion: Prenatal administration of sodium phenytoin induces limb defects in chick embryos as evident after hatching but limb anomalies are not observed on days 04 and 09 of development as investigated by stereomicroscope. We conclude that the teratogenic effect of phenytoin is time-mediated.
\end{abstract}

Key words: Chick embryos, Phenytoin, Teratogen

Authors' Contribution:

1,2 Conception, synthesis, planning of research and manuscript writing Interpretation, discussion, ${ }^{3}$ Active

participation in data collection

${ }^{4}$ Data analysis.

Cite this article. Syed HBS, Khan MY, Minhas RS. Teratogenic Effect of Sodium Phenytoin on Limb Development in Chick Embryos is Time-Mediated as Evident by Stereomicroscope. J Islamabad Med Dental Coll.2019; 8(2):60-64

\section{Introduction}

Nearly $50 \%$ patients prescribed anti-epileptic medications in Pakistan are females. ${ }^{1}$ One of such medications, sodium phenytoin is a drug commonly prescribed to pregnant women for seizures and related illnesses if the therapeutic benefit outweighs the potential risk. ${ }^{2}$ Babies born to such mothers are at an increased risk for the fetal hydantoin syndrome in which birth defects of the limbs is a consistent feature. The limb defects include digital hypoplasia, a 'finger-like' thumb, polydactyly, syndactyly, absence of palmar creases and positional limb defects (club feet). 3,4

The precise mechanism of phenytoin-induced limb defects is still unclear and several hypotheses have been proposed. ${ }^{5}$ Similar to humans, limbs appear in chick embryos as small elevations (buds) from the ventrolateral 
body wall composed of undifferentiated mesenchyme (lateral plate mesoderm) with an epithelial covering (ectoderm). The ectoderm at the distal border of the limb bud is thickened to from the apical ectodermal ridge (AER) which exerts an inductive influence on the underlying mesenchyme causing it to remain as a population of undifferentiated rapidly proliferating cells called the progress zone (PZ). As the limb bud grows the cells farther from the influence of the AER leave the $P Z$ and differentiate into cartilage and muscle, therefore the fate of the mesodermal cells is determined by the length of time spent in the PZ during limb growth. ${ }^{6}$

The current study was aimed at investigating the timemediated teratogenic role of phenytoin keeping in mind the interplay of the progress zone and AER regarding outgrowth and development of cartilaginous structures in the developing limbs. The stereomicroscope (Olympus Model SZ 40) was chosen for qualitative assessment of these parameters as it provides increased depth perception and structures can be visualized in threedimensional form. ${ }^{7}$

\section{Material and Methods}

This research was carried out in the research laboratory of the Department of Anatomy located at the Regional Center of College of Physicians and Surgeons Islamabad Pakistan, from January 2014 to January 2015. Ethical approval was given by the Research Advisory/Evaluation Committee of CPSP for Basic Medical Sciences.

A total of 180 freshly laid fertilized chicken eggs belonging to 'Egyptian Fayoumi' breed was obtained from the Poultry Research Institute (PRI), Rawalpindi. Cracked and dirty eggs were not included. All selected eggs were counted, numbered and were randomly distributed to control group (A) and experimental group (B) 90 eggs each, using the random selection table. These groups were further subdivided into subgroups 1, 2 and 3 each comprising 30 eggs. Each egg of experimental group B was administered $3.5 \mathrm{mg}$ sodium phenytoin by injection into egg albumen ${ }^{8}$ while each egg of control group was injected with equal volume of normal saline. Dosage of sodium phenytoin was calculated by using the reference from study by Singh and Shah ${ }^{9}$ and by conducting a series of pilot experiments with several preliminary doses at our laboratory. Injections were given with an insulin syringe (needle size 30-gauge $\times 8 \mathrm{~mm}$ ) and eggs were transferred to the incubator. Temperature of the incubator was kept at $38 \pm 0.5^{\circ} \mathrm{C}$, relative humidity was maintained between $60-70 \%$ and an uninterrupted electrical supply to the incubator was ensured. The eggs were rotated $1 / 2$ turn twice daily for adequate gaseous exchange and uniform distribution of heat. ${ }^{10}$

On day 04 of incubation, the embryos were gently extracted from eggs of subgroups $\mathrm{A} 1$ and B1. Each embryo was placed in a petri dish containing $70 \%$ isopropanol (2-propanol) and inspected under the stereomicroscope for the qualitative assessment of the external form and size of the limb buds. The limb bud size was recorded as small, moderate or large on the basis of Hamburger and Hamilton staging of chick embryo development.11 The observations were compared with controls and analyzed by Chi-square test according to the Statistical Package of Social Sciences (SPSS), version 16. A p-value of $\leq 0.05$ was considered statistically significant.

On day 09 of incubation, the embryos from the eggs of subgroups A2 and B2 were extracted. The cartilaginous elements were selectively stained with methylene blue and observed under the stereomicroscope to check for anomalies like duplication, truncation etc. The number of bones was counted in the proximal, middle and distal segments including the number of phalanges in the digits to check for any missing or malformed bones. The qualitative data was noted on observation sheets using the Hamburger and Hamilton staging system as a reference guide. ${ }^{11}$ The chicks belonging to subgroups $A 3$ and B3 were allowed to hatch by themselves till day 22 of incubation or assisted in hatching after day 22. The chicks of subgroup B3 were observed for gross abnormalities, lack of mobility and any abnormal behavior. Survival was noted in all the subgroups.

\section{Results}

On observation of the chick embryos of subgroup A1 under the stereomicroscope and incident illumination, each embryo was arched and had a brain (telencephalon, 
diencephalon, mesencephalon, metencephalon, myelencephalon), developing eyes, somites and heart. The developing limb buds for the wings and legs were clearly visible. The forelimb bud was identifiable as a protruding mound of tissue next to the beating heart at the level of the thoracic somites. The early hindlimb bud was also visible as a similar mass opposite the lumbosacral somites. The stereomicroscopic features of subgroup B1 were similar to that of the controls (Figure.1). The size of the limb buds was observed to be moderate in majority of the specimens and the difference in $\operatorname{limb}$ bud size between subgroups $A 1$ and $B 1$ was found to be statistically insignificant (p 0.737) (Table I).

\begin{tabular}{|c|c|c|c|c|c|c|}
\hline \multicolumn{7}{|c|}{$\begin{array}{l}\text { Table I: Comparison of Limb Bud Size (observed unde } \\
\text { stereomicroscope) between subgroups A1 and B1 and } \\
\text { Survival between all subgroups }\end{array}$} \\
\hline & \multicolumn{6}{|c|}{ Subgroups } \\
\hline & A1 & B1 & A2 & B2 & A3 & B3 \\
\hline \multicolumn{3}{|l|}{$\begin{array}{l}\text { 1: Limb bud } \\
\text { size (\%age) }\end{array}$} & & & & \\
\hline Small & 4 & 4 & & & & \\
\hline Moderate & 85 & 88 & & & & \\
\hline Large & 11 & 8 & & & & \\
\hline$P$ value & \multicolumn{2}{|c|}{0.737} & & & & \\
\hline $\begin{array}{l}\text { 2. Number } \\
\text { of surviving } \\
\text { embryos }\end{array}$ & 30 & 29 & 30 & 26 & 30 & 24 \\
\hline$P$ value & & & & & & \\
\hline
\end{tabular}

*Statistically significant

The cartilaginous pattern of the wings and legs of embryos of subgroups A2 and B2 was observed under the stereomicroscope. The methylene blue stained skeleton appeared homogeneous and well demarcated at this stage. The soft tissues were visible as translucent masses surrounding the skeletal framework. There was no duplication, truncation or missing bones. The bone count in the experimental group matched that of controls and there was no variation or deformity (Figure.2).

Survival was less in the experimental chicks as compared to the controls. This difference was statistically significant between subgroups A3 and B3 ( $p$ 0.010) (Table I). Out of the alive chicks from experimental subgroup B3, nine had wings which appeared to be smaller in size in comparison to the wings of control animals, one had abnormally positioned feet with malformed toes spread out asymmetrically and one chick showed tremors in the legs accompanied by neck torsion. All the chicks were weak and unable to stand or walk properly (Figure.3). All the dead chicks belonging to subgroup B3 exhibited a gross reduction in size whereas four of them had abdominal wall defects and one had inverted feet with malformed toes. All the chicks of control subgroup $A 3$ were alive and active without any obvious deformity.

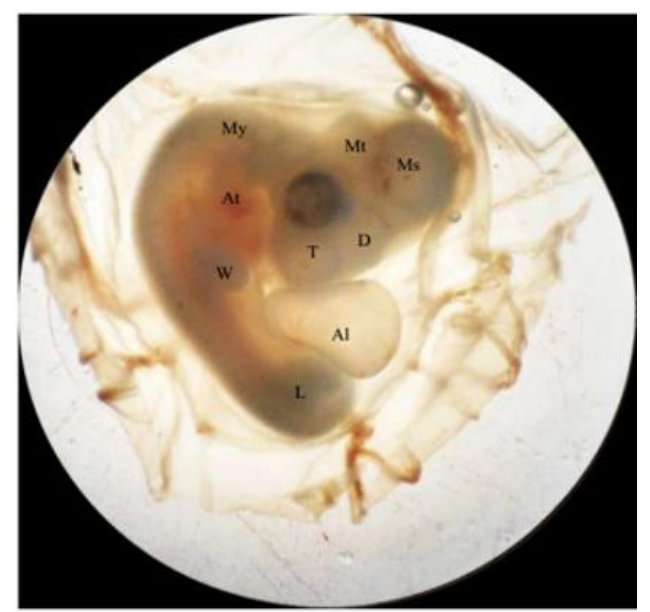

Figure 1: Photomicrograph through stereomicroscope of 04-day old chick embryo of subgroup B1; developing wing(W) and leg buds(L), Telencephalon(T), Diencephalon(D), Mesencephalon (Ms), Metencephalon (Mt), Myelencephalon (My), Atrium (At), Allantois (Al)

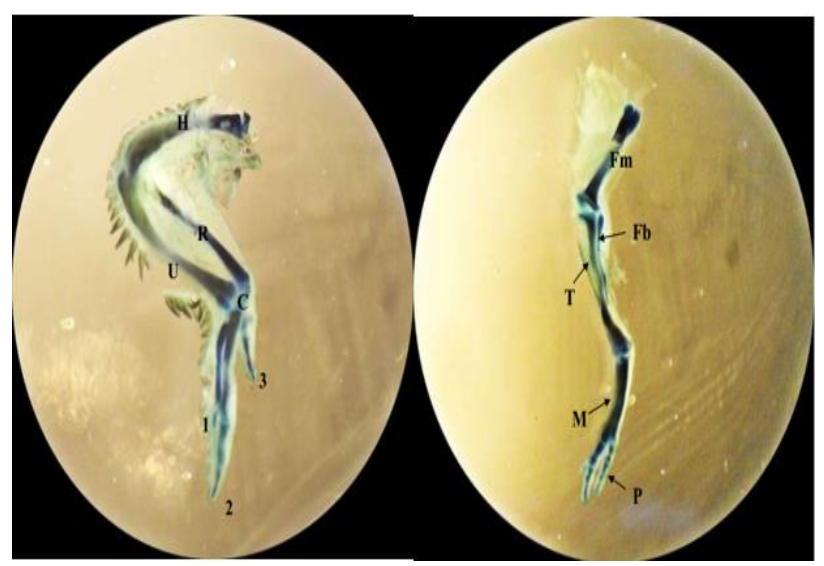

Figure 2: Photomicrograph through stereomicroscope of Methylene Blue stained 09-day old chick embryo of subgroup B2. Left- Developing wing; Humerus (H), Radius (R), Ulna (U), Carpus $(C)$, and phalanges in digits 1-3, Right-developing leg; Femur $(\mathrm{Fm})$, Tibia (T), Fibula (Fb), Metatarsus (M) and phalanges (P). 


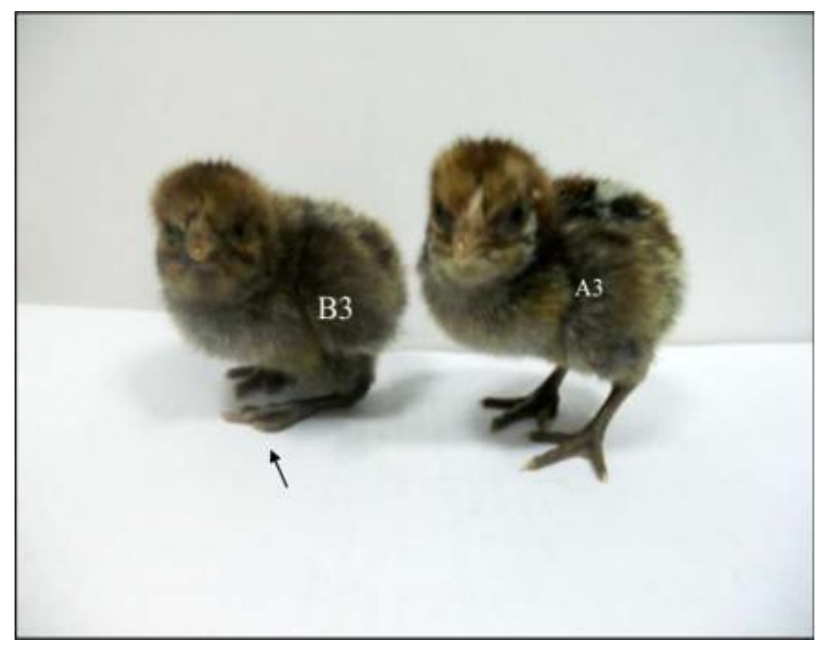

Figure 3: Photograph of newly hatched chicks belonging to experimental subgroup B3 and control subgroup A3. The one in the photograph also had inverted feet with malformed toes (arrow).

\section{Discussion}

The congenital limb defects observed in the experimental chicks in this study involved both upper and lower limbs. The live chicks showed weakness in the legs. There was a wide spectrum of limb anomalies including smaller wing size, inverted feet and malformed toes. This correlates with previous research that establishes phenytoin as a wellrecognized teratogenic agent that induces skeletal dysplasia and limb anomalies in human newborns. ${ }^{2-4}$

Limb development in human embryos begins near the end of the 4th week of intrauterine life. The critical period of limb development is from 24 to 36 days' post fertilization. ${ }^{12}$ This critical period of limb development in human embryos is represented by corresponding Hamburger-Hamilton stages 17-23 of development in the chick embryos. Therefore, day 04 of incubation (96 hours of age) which corresponds to Hamburger-Hamilton stage 23 was chosen for observation of the external form and size of the chick limb bud under the stereomicroscope in this study.11 Day 09 of incubation was chosen for observation of the cartilaginous elements in developing chick limbs because this time period corresponds to Hamburger and Hamilton stage 35 of chick development when the cartilaginous skeleton has been completely formed and can therefore, be observed for any abnormalities. ${ }^{11}$
The teratogenic effects of phenytoin have been documented in studies previously conducted on laboratory animals. Our observations are consistent with the study by Singh and Shah ${ }^{9}$ who observed the teratogenic effects of sodium phenytoin on chick embryos and found out reduced body weight, abdominal wall defects and limb defects including shortening of the wings and hypoplasia of the digits. However, the serial observation of embryos at two points before hatching is an important aspect of our research which clearly shows that the deleterious effect of phenytoin is time- mediated. It does not affect the earlier or differentiation phases of development but the later stages of growth.

Previously conducted prospective case-control cohort study by Waters ${ }^{13}$ and colleagues showed the association of maternal intake of phenytoin with an increased incidence of fetal death and anomalies. In our study, survival was less in the experimental chicks as compared to the controls. Also, the number of surviving embryos gradually decreased in the experimental groups as days of development advanced. This difference was statistically significant between the chicks of subgroups A3 and B3 further supporting the time-mediated teratogenicity of phenytoin.

Studies ${ }^{14}$ have shown that limb skeletal elements are laid down in a proximodistal sequence with the proximal elements beginning differentiation earlier than the more distal ones. The 'progress zone model' was proposed by researchers to explain skeletal patterning in vertebrate limbs. Their research describes a layer of rapidly proliferating mesenchymal cells approximately $300 \mu \mathrm{m}$ wide adjacent to and directly under the influence of the AER. Once the AER is established, it produces fibroblast growth factors (FGF4 and FGF8) which maintain the progress zone. As the limb bud grows, cells in the proximal part of the $P Z$ escape its influence and form cartilage and muscle. The cells remaining longer in the progress zone give rise to distal elements. Thus, cells residing in the $\mathrm{PZ}$ have some sort of autonomous timing mechanism. ${ }^{15}$ Our study seems to support this hypothesis that the undifferentiated zone of the limb bud are more prone to teratogenic effects of phenytoin as evident by the distal limb defects in the B3 experimental group. 
In our study, upon close inspection with the stereomicroscope, the cartilage pattern and number of bones was normal with no missing or duplicated pieces in the experimental chick embryos of subgroup B2. A possible explanation for this may be that the dose of phenytoin administered in this study was insufficient to produce any visible anomaly at this earlier stage of differentiation. Similar to limb bud size, the cartilage pattern did not show any irregularities further supporting the conclusion that the teratogenic effect of sodium phenytoin must be time mediated.

This study warrants precaution in administering sodium phenytoin and similar antiepileptic medications to pregnant ladies as the birth defects particularly limb anomalies might not be evident until much later stages of development. The main limitation of our study was that the stereomicroscope allows viewing a relatively large object in three dimensions, but provides low magnification.

\section{Conclusion}

Exposure of chick embryos to sodium phenytoin produces visible limb defects after hatching. No abnormality of limb morphogenesis is observed during developmental days 04 and 09 when inspected under the stereomicroscope. Therefore, we conclude that the teratogenic effect of sodium phenytoin is time-mediated and the limb defects were induced at the maturation stage instead of differentiation.

\section{References}

1. Mazhar F, Shamim S, Malhi SM. Drug utilization evaluation of antiepileptics in three selected multidisciplinary teaching hospitals of Pakistan. Int J Pharm Pharm Sci 2014; 6(5): 5966.

2. Tomson T, Battino D. Teratogenic effects of antiepileptic drugs. The Lancet Neurol 2012; 11(9): 803-13. doi: 10.1016/S1474-4422(12)70103-5

3. Singh R, Kumar N, Arora S, Bhandari R, Jain A. Fetal hydantoin syndrome and its anaesthetic implications: a case report. Case Rep Anesthesiol 2012. doi: 10.1155/2012/370412

4. Singh A, Bhatia HP, Mohan A, Sharma N. Fetal hydantoin syndrome: A case report. J Indian Soc Pedod Prev Dent. $2016 ; 34(1): 92-5$.

5. Etemad L, Moshiri M, Moallem SA. Epilepsy drugs and effects on fetal development: Potential mechanisms. J Res Med Sci 2012; 17(9): 876-81. PMID: 23826017

6. Sadler TW. Langman's Medical Embryology. 12th ed. Philadelphia: Lippencott William and Wilkins 2012.

7. Jia S, Chen D, Wang D, Bao X, Tian X. Comparing marginal microleakage of three different dental materials in veneer restoration using a stereomicroscope: an in vitro study. BDJ open. 2017; 3: 16010. doi: 10.1038/bdjopen.2016.10

8. Ahangari YJ, Hashemi SR, Akhlaghi A, Atashi H, Esmaili Z, Ghorbani M, et al. Effect of in ovo injection of royal jelly on posthatch growth performance and immune response in broiler chickens challenged with newcastle disease virus. Iran J Appl Anim Sci 2013; 3(1): 201-06.

9. Singh $M$, Shah GL. Teratogenic effects of phenytoin on chick embryos. Teratology. 1989;40(5):453-8.

10. Tona K, Onagbeasan O, Bruggeman V, Mertens K, Decuypere $E$. Effect of turning duration during incubation on embryo growth, utilization of albumen and stress regulation. Poult Sci. 2005; 84(2): 315-20.

11. Hamburger $V$, Hamilton $H L$. A series of normal stages in the development of the chick embryo. 1951. Dev Dyn. 1992; 195(4): 231-72. doi: 10.1002/aja.1001950404

12. Moore K. L, T.V.N Persaud, Torchia M. G. The developing human: Clinically oriented embryology. 10th Ed. Philadelphia, PA: Elsevier 2016.

13. Waters $\mathrm{CH}$, Belai $\mathrm{Y}$, Gott PS, Shen P, De Giorgio CM. Outcomes of pregnancy associated with antiepileptic drugs. Arch Neurol. 1994;51(3):250-53.

14. Fernandez-Teran M, Ros MA. The Apical Ectodermal Ridge: morphological aspects and signalling pathways. Int J Dev Biol. 2008; 52(7): 857-71. doi: 10.1387/ijdb.072416mf

15. Molineux AC, Maier JA, Schecker T, Sears KE. Exogenous retinoic acid induces digit reduction in opossums (Monodelphis domestica) by disrupting cell death and proliferation, and apical ectodermal ridge and zone of polarizing activity function. Birth Defects Res A Clin Mol Teratol. 2015;103(3):225-34. 